\title{
Notfalldienst - unzumutbare gesetzliche Regelungen als Chance für den Ärzteverband?
}

Patrick Stach ${ }^{a}$, Daniel Bleuer ${ }^{b}$

a Dr. iur., Rechtsanwalt

b M. A., HSG in Law \& Economics,

St. Gallen
Korrespondenz:

RA Dr. iur. Patrick Stach

Stach Rechtsanwälte und Notare

Dufourstrasse 121

CH-9001 St. Gallen

Tel. 0712787828

stach[at]stach.ch
Die Standesorganisationen nehmen im Bereich der Organisation des Notfalldienstes vorwiegend öffentliche Interessen wahr und regeln die Erfüllung öffentlicher Aufgaben.

Die mangelhafte gesetzliche Ausgestaltung erlaubt ihnen aber nicht, die entsprechenden Pflichten im Einzelfall effektiv durchzusetzen. Auch in finanzieller Hinsicht sehen sich die Standesorganisationen vor grosse Herausforderungen gestellt. Rechnungen von Privaten bleiben unbezahlt und finanzielle Beiträge der öffentlichen Hand fehlen. Der Zustand erweist sich als unzumutbar.

Notwendige gesetzliche Anpassungen bieten gleichsam Chancen, um diese Zustände zu beheben und die bedeutsame Frage der Entschädigung durch das Gemeinwesen einer gesetzlichen Regelung zuzuführen.

\section{Ausgangslage}

Die Ausgestaltung des ärztlichen Notfalldienstes war lange Zeit ausschliesslich privater Initiative überlassen. Ärztinnen und Ärzte schlossen sich zu privaten Standesorganisationen zusammen und übernahmen die Organisation des Notfalldienstes, der als willkommene zusätzliche Einnahmequelle und als Möglichkeit zur Vergrösserung des Patientenkreises galt [1].

Diese Ansicht hat sich in letzter Zeit jedoch stark gewandelt. Heute wird das Erbringen von Notfalldienst überwiegend als Last empfunden. Immer mehr Ärzte lassen sich vom Notfalldienst «dispensieren», was dessen Organisation erheblich erschwert. Schwangerschaft, Mutterschaft und Kleinkinderziehung weiblicher Kolleginnen in freier Praxis reduziert zudem die Anzahl Dienstleistender, weil gerade in diesen Fragen kulante Dispenslösungen gesucht werden müssen. Aber auch in finanzieller Hinsicht zeigt sich die Sachlage alles andere als rosig. Finanzielle Beiträge der Gemeinden fehlen, und die Zahlungsmoral der Patienten erweist sich oftmals als mangelhaft. Alleine in der Stadt St. Gallen blieben bis heute Rechnungen im Umfang von 1,5 Millionen Franken unbezahlt [2].

Die faktischen Veränderungen der vergangenen Zeit haben aber nur marginal zu gesetzgeberischen Anpassungen geführt. Wird die rechtliche Ausgestaltung des Notfalldienstes einer genaueren Betrachtung zugeführt, werden vielmehr grundlegende
Service de garde - des réglementations légales jugées inadmissibles peuvent-elles être une chance pour la société de médecine?

Les organisations professionnelles chargées de l'organisation du service de garde assument en premier lieu des tâches d'intérêt public et en règlent la réalisation. Le cadre légal lacunaire ne leur permet toutefois pas dans tous les cas de remplir efficacement leur cahier des charges. Sur le plan financier aussi, elles sont confrontées à de gros défis, car les factures des privés demeurent impayées et les deniers publics manquent: une situation intolérable. Les adaptations légales nécessaires offrent pour ainsi dire une chance de résoudre ce problème et d'inscrire dans la loi la question de la rémunération par la collectivité.

Mängel und Versäumnisse offenkundig, welche die Organisation des Notfalldienstes zusätzlich erschweren. Dass die heutige Rechtslage sogar das Prädikat «unzumutbar» verdient, soll nachfolgend aufgezeigt werden.

\section{Gesetzliche Ausgestaltung der Notfalldienstpflicht}

Am 1. September 2007 ist das Bundesgesetz über die medizinischen Medizinalberufe (MedBG) in Kraft gesetzt worden. Es auferlegt Personen unter anderem die Pflicht, gemäss Art. 40 lit. g) nach Massgabe der kantonalen Vorschriften in Notfalldiensten mitzuwirken.

Die Kantone haben in Ausführung dieser Vorschrift meist die Standesvereinigungen (Standesorganisationen) mit der Organisation des Notfalldienstes betraut. In St. Gallen haben sich die Personen, die 
einen medizinischen Beruf ausüben, am Notfalldienst ihrer Standesorganisation zu beteiligen. Die Standesorganisationen werden gleichsam - im Sinne einer Gesetzgebungskompetenz - ermächtigt, den Notfalldienst durch Reglement zu regeln [3].

Damit wurde rechtlich umgesetzt, was bereits seit langem galt, nämlich die Verantwortung der Standesorganisationen für den Notfalldienst. Die vermeintlich geringfügigen gesetzlichen Änderungen wurden ohne grosse Diskussion in Gesetzen, aber teilweise auch nur in Verordnungen, verankert. Dass man sich deren tiefgreifenden Folgen nicht bewusst war, wird bei einer genaueren Analyse deutlich.

\section{Der Notfalldienst als öffentliche Aufgabe}

Gemäss Art. 15 lit. a) und b) der St. Galler Kantonsverfassung setzt sich der Staat zum Ziel, dass die Bevölkerung zu für sie tragbaren Bedingungen eine ausreichende Gesundheitsversorgung erhält und eine wirksame und breitgefächerte Gesundheitsvorsorge und Gesundheitserziehung bestehen. Ähnlich lautende Regelungen finden sich auch in anderen Kantonen [4].

Dazu gehört auch der Notfalldienst. Auf Verordnungsstufe wird den Ärzten in St. Gallen die Pflicht auferlegt, an Notfalldiensten teilzunehmen. Hierdurch soll die medizinische Versorgung der Bevölkerung ausserhalb der üblichen Sprechstunden sichergestellt werden, wobei der Notfalldienst regelmässig der keinen Aufschub duldenden Behandlung von Erkrankungen dient [5].

Die Einrichtung eines Notfalldienstes dient dem öffentlichen Interesse an der Gewährleistung der Gesundheit der Bevölkerung. Neben einer ortsnahen Versorgung soll sie auch die Notfallstationen der Spitäler entlasten, wodurch diese insbesondere für schwerwiegende Fälle freigehalten werden. Die anwendbaren Rechtssätze - insbesondere auch die Reglemente der Standesvereinigungen - nehmen somit vorwiegend öffentliche Interessen wahr und regeln die Erfüllung öffentlicher Aufgaben. Insoweit handelt es sich um öffentliches Recht und beim Notfalldienst um eine öffentliche Aufgabe [6].

\section{Mangelhafte gesetzliche Umsetzung der Notfalldienstpflicht}

Wer staatliche Aufgaben (öffentliche Aufgaben) wahrnimmt, ist an die Grundrechte gebunden und verpflichtet, $\mathrm{zu}$ ihrer Verwirklichung beizutragen (Art. 35 Abs. 2 BV). Die Delegation von öffentlichen Aufgaben oder von Verwaltungsbefugnissen an einen Privaten hebt diese verfassungsrechtliche Pflicht nicht auf [7].

Die Übertragung von Verwaltungsaufgaben auf Private ist daher nur zulässig, wenn sie auf gesetzlicher Grundlage beruht, die Privaten der Aufsicht des Staates unterstehen und gewährleistet ist, dass die Privaten die Verfassung und insbesondere die Grundrechte beachten [8].

Da die kantonalen Bestimmungen die Detailregelungen über den Notfalldienst in den überwiegenden Fällen zudem den Standesvereinigungen übertragen, liegt nicht nur eine Übertragung von Verwaltungsaufgaben auf Private vor, sondern gleichsam eine Übertragung von Rechtsetzungskompetenzen auf private Organisationen.

\section{Die heutige Rechtslage verdient so- gar das Prädikat «unzumutbar».}

Die Delegation von Rechtsetzungsbefugnissen an nichtstaatliche, in der Verfassung nicht verankerte Organisationen, stellt einen erheblichen Eingriff in die von der Verfassung vorgesehene Kompetenzordnung im Bereich der Rechtsetzung dar. Eine solche ist nach herrschender Lehrmeinung nur zulässig, wenn sich diese auf einer Grundlage in einem Erlass der Gesetzesstufe stützen kann und sich auf Fragen untergeordneter, vor allem technischer Natur beschränkt [9].

Eine Untersuchung der kantonalen Normen zeigt, dass die Übertragung von Verwaltungsaufgaben sowie entsprechender Rechtsetzungskompetenzen häufig nur auf Verordnungsstufe basieren. Im Kanton St. Gallen beispielsweise fusst die Pflicht der Ärzte, sich den Standesorganisationen anzuschliessen, sowie die Befugnis dieser Organisationen, ein entsprechendes Reglement zu erlassen, lediglich auf Art. 13 der Verordnung über die Ausübung der medizinischen Berufe. Diese gesetzliche Umsetzung erweist sich als ungenügend.

\section{Problematik bei der Erhebung von Ersatzabgaben}

In den kantonalen Reglementen der Standesvereinigungen wird häufig die Möglichkeit vorgesehen, sich von der Notfalldienstleistungspflicht dispensieren zu lassen. Hierfür ist eine Dienstpflichtersatzabgabe auszurichten.

Wer zur Erfüllung einer Staatsaufgabe sachlich, örtlich und funktionell zuständig ist, ist auch zuständig, damit verbundene Verwaltungsverhältnisse durch Verfügung zu regeln; Verwaltungsbefugnis schliesst Verfügungsbefugnis ein [10]. Mit der Übertragung einer öffentlichen Aufgabe an eine verwaltungsexterne Einheit sind die vom Übertragungsakt abgedeckten hoheitlichen Befugnisse verbunden, die zur Erfüllung der übertragenen öffentlichen Aufgaben erforderlich sind [11].

Bei der Notfalldienstleistungspflicht handelt es sich um eine öffentlich-rechtliche Pflicht. Bei einer 


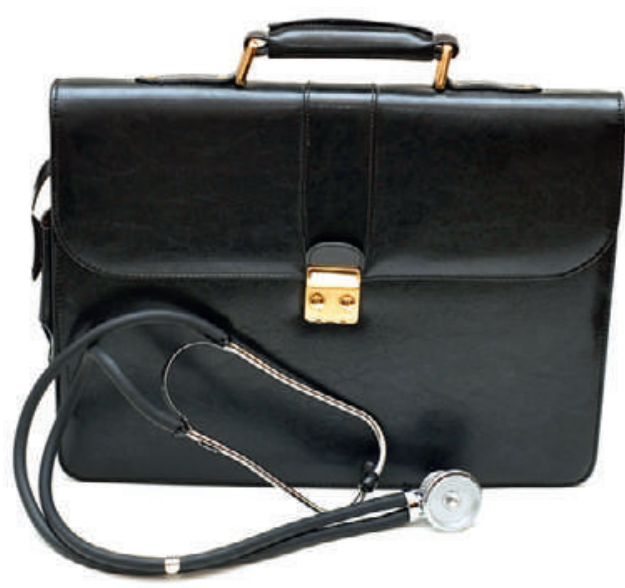

Der Einsatz im Notfalldienst wird immer unbeliebter, zumal die Zahlungsmoral vieler Patienten schlecht ist. In St. Gallen sind Rechnungen in Höhe von 1,5 Millionen Franken ausstehend.

Entschädigung, die zur Entbindung dieser Pflicht geleistet werden muss, handelt es sich demnach um eine öffentliche Abgabe in der Form der Ersatzabgabe.

An die Erhebung von öffentlich-rechtlichen Abgaben werden klare Voraussetzungen gesetzt. So bestimmt Art. 127 Abs. 1 BV für alle Abgaben, dass zumindest der Kreis der Abgabepflichtigen, der Gegenstand der Abgabe und die Höhe der Abgabe in den Grundzügen in einem Gesetz geregelt werden müssen [12].

\section{Ungenügende Finanzierung}

So weit Private öffentliche Aufgaben erfüllen, werden diese in der Regel vom Gemeinwesen oder von den Privaten, die ihre Dienste in Anspruch nehmen, entschädigt [14].

Im Bereich des Notfalldienstes zeigen sich aber gerade hinsichtlich der Entschädigung durch die Privaten grosse Schwierigkeiten. Oftmals wird die Behandlungspflicht im Rahmen des Notfalldienstes (missbräuchlich) beansprucht, um zu einer «kostenlosen» Behandlung zu gelangen. Alleine in der Stadt St. Gallen sind Rechnungen im Umfang von 1,5 Millionen Franken ausstehend.

Eine staatliche Unterstützung scheint notwendig. Den Kantonen verbleibt aber in der Regel ein grosser Ermessensspielraum. Nach Massgabe der Verfassung des Kantons St. Gallen kann das Gemeinwesen Private unterstützen, sofern diese Aufgaben von öffentlichem Interesse wahrnehmen [15]. Ein klagbarer Anspruch auf finanzielle Unterstützung steht den Standesvereinigungen somit in aller Regel nicht zu [16].

\section{Unzumutbare Situation der Standesvereinigungen}

Welche unzumutbaren Folgen eine mangelhafte gesetzliche Umsetzung zeitigen kann, wird am Beispiel des Kantons St. Gallen deutlich. Die Verpflichtung, sich den Standesorganisationen anschliessen $\mathrm{zu}$ müssen, basiert ausschliesslich auf Verordnungsstufe. Dies genügt nach der hier vertretenen Ansicht dem Legalitätsprinzip nicht. Darauf beruhende Grundrechtseingriffe erweisen sich als nicht ge-

\section{Die Ärztegesellschaften haben im Rahmen der Revision die Möglichkeit, eine gesetzliche Regelung der finanziellen Entschädigung einzufordern.}

Für die Erhebung einer Ersatzabgabe durch die Standesvereinigungen bedarf es einer klaren gesetzlichen Grundlage. Die Ausgestaltung der Form, Art und Höhe der Abgabe in einem Reglement erweist sich als ungenügend. Die Erhebung einer entsprechenden Abgabe verstösst gegen das verfassungsmäs sige Legalitätsprinzip von Art. 127 Abs. 1 BV und liesse sich u. E. nicht durchsetzen.

Im Kanton St. Gallen zeigt sich die Rechtslage besonders problematisch, denn es besteht weder eine gesetzliche Grundlage für die Erhebung von Ersatzabgaben noch eine ausreichende Delegation von Rechtsetzungsbefugnissen an die Standesvereinigungen. Ein unzumutbarer Zustand für viele Standesorganisationen. So finanziert sich beispielsweise die Standesorganisation der Stadt St. Gallen beinahe ausschliesslich durch Ersatzabgaben. Fallen diese weg, so könnte der Notfalldienst nicht mehr gewährleistet werden [13]. rechtfertigt und demzufolge als widerrechtlich [17].

Die Verpflichtung, sich den Standesorganisationen anschliessen $\mathrm{zu}$ müssen, führt $\mathrm{zu}$ einer Grundrechtseinschränkung im Bereich der freien Wahl der Organisationsform, der Freiheit unternehmerischer Betätigung und der freien Wahl der Geschäftsbeziehungen. Aber auch die Vertragsfreiheit wird durch die Pflicht, Notfalldienst nach Massgabe der Reglemente der Standesorganisationen zu leisten, eingeschränkt [18].

Fehlt es bei der legislatorischen Umsetzung an einer genügenden gesetzlichen Grundlage, so haben Ärzte diese Einschränkung nicht zu dulden. Ihnen steht es zu, den Notfalldienst selber zu organisieren oder für eine andere zweckmässige Organisation zu sorgen. Das führt zum unbefriedigenden Ergebnis, dass sich diese zwar am Notfalldienst der Standesorganisationen beteiligen können, hierzu aber nicht gezwungen werden dürfen [19]. 


\section{Mangelhafte gesetzliche Umsetzung als Chance}

Eine Gesetzesrevision auf Kantonsebene ist unabdingbar, um die Rechte und Pflichten im Rahmen der Notfalldienstleistung in eine klare Form zu giessen. Die Standesorganisationen erfüllen heute eine wichtige öffentliche Aufgabe. Die gesetzliche Ausgestaltung erlaubt ihnen aber nicht, die entsprechenden Pflichten im Einzelfall durchzusetzen. Diese Situation ist gerade hinsichtlich der grossen Bedeutung des Notfalldienstes äusserst unbefriedigend und führt zu einer grossen Rechtsunsicherheit.

Um den Standesorganisationen wirksame Instrumente zur Verfügung zu stellen, bedarf es gesetzlicher Anpassungen. Dieser Prozess bietet gleichsam Chance, um auch die bedeutsame Frage der Entschädigung zu regeln. Obwohl den Standesorganisationen in den überwiegenden Fällen kein gesetzlicher Anspruch auf finanzielle Entschädigung durch das Gemeinwesen zusteht, haben die Ärztegesellschaften im Rahmen dieser Revision die Möglichkeit, auf den Gesetzgeber einzuwirken. Die (neuen) gesetzlichen Regelungen sollten dabei nicht nur die Grundlagen der Notfalldienstpflicht gestalten, sondern den Standesorganisationen auch einen klagbaren Anspruch auf Entschädigung einräumen.

\section{Literatur}

1 Poledna, Stoll. Ärztlicher Notfalldienst: Pflicht oder Recht des Arztes? AJP. 2005;1367.

2 Angaben des Ärztevereins der Stadt St. Gallen.

3 Vgl. Art. 13 Abs. 2 der Verordnung über die Ausübung der medizinischen Berufe.

4 Vgl. Art. 13 der Kantonsverfassung in Zürich (SR 131.211); Art. 100 der Kantonsverfassung in Solothurn (SR 131.221).

5 Fellmann W. In: Schaffhauser, Kieser, Poledna (Hrsg.). Das neue Medizinalberufsgesetz. 2008; S. 114-6.
Etter B. Medizinalberufsgesetz. Bern: Stämpflis Handkommentar; 2006. N 40 ff. zu Art. 40 MedBG.

6 Vgl. BGer 2C.807/2010, E. 2.6; Poledna, Stoll, a.a.O., S. 1372 .

7 Vgl. BGE 127 I 84; 103 Ia 544.

8 Häfelin/Haller, Schweizerisches Bundesstaatsrecht, 5. Aufl., Zürich 2001, N 1509.

9 Häfelin, Haller, a.a.O., N 1890.

10 Tschannen, Zimmerli, Müller. Allgemeines Verwaltungsrecht, 3. Aufl., \$28 Rz. 19ff; vgl. auch BGE 121 II 454; BGer 2C_715/2008.

11 BGE 129 II 331; vgl. BGer 2C_715/2008.

12 Das Bundesgericht überprüfte die Regelung des Gesundheitsgesetzes im Kanton Thurgau und hat die Rechtssetzungsdelegation zur Erhebung von Ersatzabgaben als ungenügend angesehen und deren Anwendung versagt (BGer 2C_807/2010).

13 Angaben des Ärztevereins der Stadt St. Gallen.

14 Häfelin, Müller, Uhlmann, Allgemeines Verwaltungsrecht, Zürich 2006, 5. Aufl., N 1515.

15 Vgl. Art. 24 Abs. 2 KV SG.

16 Es kann diesbezüglich nicht den Ausführungen von Simon Graf gefolgt werden. Den Gemeinden steht hinsichtlich der finanziellen Unterstützung grosses Ermessen zu. Vielmehr handelt es sich um ein generell gültiges Prinzip, wonach der Staat die Durchführung von individuellen Pflichten mit exekutorischen Sanktionen erzwingen kann. Werden Pflichten nicht erfüllt, so sorgt die politische Gemeinde auf Kosten des Primärverpflichteten für deren Durchsetzung (vgl. Graf S. Die Pflicht zur Leistung von Notfalldienst durch Ärzte in der Praxis. Schweiz Ärztezeitung. 2012;93(5):170-3.

17 Vgl. Art. $36 \mathrm{BV}$.

18 Graf S. Die Pflicht zur Leistung von Notfalldienst durch Ärzte in der Praxis. Schweiz Ärztezeitung. 2012;93(5):171.

19 Die Mitglieder des FMH oder der Ärztegesellschaft des Kantons St. Gallen sind aber immerhin statutarisch verpflichtet, sich am Notfalldienst des jeweiligen Regionalvereins zu beteiligen (vgl. Art. 40 der Standesordnung FMH) 